\title{
The Treatment of Partial-Thickness Burns with a Hydroconductive Wound Dressing: Clinical and Mechanistic Effects
}

\author{
David J. Smith ${ }^{1}$, Rachel A. Karlnoski ${ }^{1,2}$, Ami Patel ${ }^{2}$, \\ C. Wayne Cruse ${ }^{1}$, Kimberly S. Brown $^{3}$, Martin C. Robson ${ }^{{ }^{*} \#}$ \\ ${ }^{1}$ Department of Surgery, University of South Florida, Tampa, USA \\ ${ }^{2}$ Florida Gulf-to-Bay Anesthesiology Associates, LLC, Tampa, USA \\ ${ }^{3}$ Tampa General Hospital Burn Center, Tampa, USA \\ Email: "robsonmd1@gmail.com
}

Received March 17, 2013; revised April 19, 2013; accepted April 28, 2013

Copyright (c) 2013 David J. Smith et al. This is an open access article distributed under the Creative Commons Attribution License, which permits unrestricted use, distribution, and reproduction in any medium, provided the original work is properly cited.

\begin{abstract}
Objectives: Edema in partial-thickness burn wounds can decrease tissue perfusion, increase tissue ischemia, and deepen the burn injury. We report the results of a clinical trial comparing the effectiveness of a hydro-conductive dressing to our standard burn dressing at removing edema fluid from partial-thickness burns and present the proposed mechanisms of action of the hydroconductive dressing. Methods: An internally controlled comparison of two wound dressings was performed on 10 patients with non-contiguous partial-thickness burns. Each patient served as his/her own control. One burn was treated with our standard burn dressing and the other with hydroconductive dressing. Dressings were weighed prior to application, removed at 24 and 48 hours, weighed, and new pre-weighed dressings applied. Weight gain of each dressing at 24 and 48 hours was determined. Statistics were applied using the Student's paired T-test. The VAS pain scale was measured prior to, during, and after each dressing change. Results: At 24 hours, the hydroconductive dressing had increased $85.6 \% \pm 29.3 \%$ in weight compared with $61.3 \% \pm 32.7 \%$ for the control $(\mathrm{P}=$ $0.053)$. For the second 24 hour period, the respective numbers were statistically significantly different at $59.7 \% \pm$ $23.4 \%$ vs. $34.2 \% \pm 19.1 \%(\mathrm{P}=0.038)$. Averaging the weight gain over the two dressing periods demonstrated that the differences were highly statistically significant as the hydroconductive dressing increased in weight by $71.0 \% \pm 20.3 \%$ compared with $44.5 \% \pm 17.4 \%$ for the gauze dressing $(\mathrm{P}=0.005)$. VAS scores revealed no statistical differences. Mechanisms of action included capillary, hydroconductive, and electrostatic actions. Conclusions: A hydroconductive dressing designed to draw off excessive wound fluid removes more wound edema fluid than standard burn gauze dressings.
\end{abstract}

Keywords: Burn Wound; Edema

\section{Introduction}

Edema is a hallmark of partial-thickness burn wounds. It is maximum 12 - 24 hours post-injury and persists for 48 72 hours. Edema can decrease tissue perfusion, increase tissue ischemia, and deepen the burn injury. In functional areas such as the hands, persistent edema fluid can increase tissue fibrosis and limit range of motion. A new class of wound dressing, hydroconductive dressings, has been introduced that has the properties to draw off excessive fluid, tissue bacteria, and cytokines deleterious to

*Dr. Robson is a medical and research advisor to SteadMed Medical, LLC.

${ }^{\#}$ Corresponding author. wound healing [1]. The purpose of this study was to compare the effectiveness of this hydroconductive dressing to our standard burn wound dressing at removing edema fluid from partial-thickness burn wounds and to present the mechanisms of action of the hydroconductive dressing.

\section{Methods}

An IRB-approved, internally controlled, comparison of two wound dressings was performed on 10 adult patients with partial-thickness burns of scald or flame etiology. Only patients that were admitted to the burn center and were predetermined to require burn dressings for partial 
thickness burns and met all study criteria were enrolled in the study. The inclusion and exclusion criteria for the study are presented in Tables $\mathbf{1}$ and $\mathbf{2}$.

Two non-contiguous burns were chosen for each patient, allowing each patient to serve as his/her own control. After receiving informed consent, the two burn wounds were debrided of all blisters. A thin layer of Silvadene cream was then applied to each burn wound as is the standard treatment protocol at our Burn Center. One burn was chosen to be treated with our standard burn fluffed gauze dressing and the other with the hydroconductive dressing, Drawtex (SteadMed Medical LLC, Fort Worth, TX). Allocation of treatment of wounds sites was done at random, using a pre-determined random assignment of treatments to the 2 defined wound regions (Figure 1). Randomization scheme was achieved using a computer-generated list (generated by the biostatistician). Wound regions were initially labeled A and B by the physician, and then an envelope was opened to indi cate which treatment was assigned to $\mathrm{A}$ and which to $\mathrm{B}$.

Both the standard burn gauze dressing and a single layer of Drawtex were weighed prior to application.

Table 1. Inclusion criteria.
1) Patients with 2 non-contiguous second degree burns;
2) Wound sizes of $100-320 \mathrm{~cm}^{2}$;
3) TBSA up to $25 \%$;
4) Burn of thermal origin;
5) Burns present on trunk and extremities;
6) Both genders with an age 18 - 65 years at randomization;
7) Signed informed consent.

Table 2. Exclusion criteria.

1) Burns located on Head, neck, or hands;

2) Burns of chemical and electrical origin;

3) Clinically infected burn (as judged by the investigator);

4) Patients with necrotizing leucocytic vasculitis or pyoderma gangrenosa;

5) Diagnosed underlying disease(s) (e.g. HIV/AIDS, cancer and severe anemia) judged by the investigator to be a potential interference in the treatment;

6) Patients with insulin dependent diabetes mellitus;

7) Patients treated with systemic glucocorticosteroids, except patients taking occasional doses or doses less than $10 \mathrm{mg}$ prednisolone/day or equivalent;

8) Use of immunosuppressive agents, radiation or chemotherapy within the past 30 days;

9) Known allergy/hypersensitivity to any of the components of the investigation products;

10) Patients with physical and/or mental conditions that are not expected to comply with the investigation;

11) Participation in other clinical investigation(s) within 1 month prior to and at the start of the investigation;

12) Pregnancy.

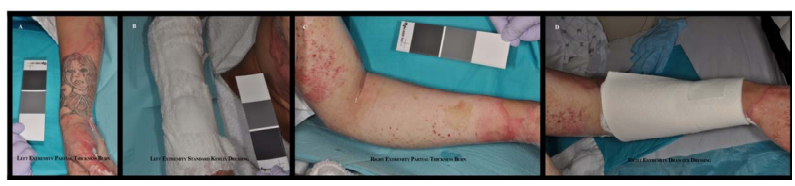

Figure 1. Patient with partial-thickness burns to right upper extremity treated with standard burn gauze dressing and left upper extremity treated with hydroconductive dressing.

Every 24 hours the dressings were changed. Both study dressings were weighed after removal on day 1 . The study wounds were redressed in the same manner on the following day. In order to compare the dressings, their respective weights were differentiated. The differences in weight were obtained at 24 and 48 hours. The same practitioner attempted to change the dressings; however, it was not possible for all cases. To account for this eventuality, all burn center nurses were similarly trained for dressing changes. Percentage weight gain of each dressing at 24 hours and 48 hours was determined. Statistics were applied using the Student's paired T-test. All other non-study burn sites were treated with our standard of care. Although for superficial partial-thickness burns it is not necessary that topical antibacterial agents be used, our standard of care is a layer of Silvadene covered with bulky burn gauze dressing. The wound dressings are changed every 24 hours but may be more frequent depending upon volume of drainage or the physical condition of the dressing.

All patients received analgesia according to our standard pain protocol. The standard pain management regimen at our burn center is: $10 \mathrm{mg}$ oxycodone or $2 \mathrm{mg} \mathrm{Di}$ laudid by mouth approximately 1 hour before dressing change. Alternatively, $10 \mathrm{mg}$ Morphine IV may be given approximately 10 minutes before dressing change. Pain was assessed at each dressing change using a standard Visual Analog Pain Scale (VAS) rated $1-10$ on a $10 \mathrm{~cm}$ line. Pain was assessed 30 minutes before the dressing change, during the dressing change, and 30 minutes after the dressing change. If there were multiple non-study wound sites, study sites were changed first for more accurate assessment of pain.

Sample size selection was based on the feasibility of completing a small number of subjects in a pilot study to be used in the development of a larger study including sample size determination with power analysis. If the data demonstrated statistical significance, no additional subjects would be required.

\section{Results}

At the first dressing change, the hydroconductive dressing had increased $85.6 \% \pm 29.3 \%$ in weight compared with $61.3 \% \pm 32.7 \%$ for the control standard burn dressing $(P=0.053)$. This trend was not statistically signifi- 
cant. For the second 24-hour-period, the differences were statistically significant with respective numbers of $59.7 \%$ $\pm 23.4 \%$ for the hydroconductive dressing vs. $34.2 \% \pm$ $19.1 \%$ for the control $(\mathrm{P}=0.038)$. Averaging the weight gain in the dressings over the two dressing periods demonstrated highly statistically significant differences in that the hydroconductive dressing increased in weight by $71.0 \% \pm 20.3 \%$ compared with $44.5 \% \pm 17.4 \%$ for the gauze dressing $(\mathrm{P}=0.005)$. The hydroconductive dressing removed a statistically significant greater amount of edema fluid from the burn wounds.

The VAS scores were not statistically significantly different for the two dressings at any time point prior to, during, or following dressing changes. Therefore, the additional effectiveness of drawing off edema fluid was not associated with increased pain or stinging for the hydroconductive dressing.

\section{Discussion}

The ideal burn dressing should be one that maintains a moist pH-balanced wound, absorbs exudates, limits infections, minimizes disturbances of healing tissue beneath the dressing, reduces pain to the patient, and reduces dressing changes [2]. The standard practice for partial thickness wounds depends on the depth of the wound and the co-morbidities that the patient possesses. For superficial partial-thickness burns it is not necessary that topical antibacterial agents be used. However, at Tampa General Hospital the standard of care is the use of Silvadene ${ }^{\circledR}$ covered with a bulky gauze dressing. The frequency with which the dressings are changed is arbitrary and dictated by the volume of drainage or the physical condition of the dressing.

Drawtex, a new hydroconductive wound dressing has been demonstrated to draw off excessive wound exudate, wound debris, bacteria, and MMPs [1,3-5]. The dressing is composed of three layers utilizing a proprietary construction with Leva Fiber Technology. The question remains as to the mechanisms responsible for the actions seen with Drawtex treatment of a wound. It appears that the mechanisms are due to a combination of physical actions: capillary action, hydroconductive action, and electrostatic action that work together. This combination of actions is unique for a wound dressing. To understand these mechanisms, each will be briefly discussed.

Capillary action is the ability of a liquid to flow in narrow spaces without the assistance of, and in opposition to external forces like gravity [6]. It is caused by the attracttion of molecules of the liquid to the molecules of the solid [7]. This effect can be seen in the drawing up of liquids into porous materials such as certain textiles. Molecules of water are naturally attracted to each other and form temporary hydrogen bonds with each other, but they are also attracted in a similar way to other molecules, called hydrophilic molecules, such as those in the fibers of a textile [7]. These forces can draw water upward against the force of gravity to a certain degree. The small pores of the dressing, Drawtex, act as small capillaries, causing it to absorb a comparatively large amount of fluid (Figure 2).

Hydroconductive action is the movement of water in both vertical and horizontal directions. This action is controlled by Darcy's Law that defines the ability of a fluid to flow through porous media [8,9]. Fluid can move from wetter to drier, even against gravity (Figure 3). A subclassification of hydroconductive action is transpiration and depends on the ability to have evaporation from the edges or surface of the porous material which is moving the fluid [10]. This hydroconductive action allows Drawtex to lift, hold and transfer the wound exudate both vertically and horizontally into the dressing.

Electrostatic action is due to the attraction or repulsion between two electrically charged bodies. Although the capillary action and hydroconductive action explain the drawing off of exudate and particles contained in the exudate, they do not account for Drawtex's ability to draw bacteria and deleterious cytokines such as proteases from a wound into the dressing. This mechanism is due to the electrostatic action between the bacteria and pro-

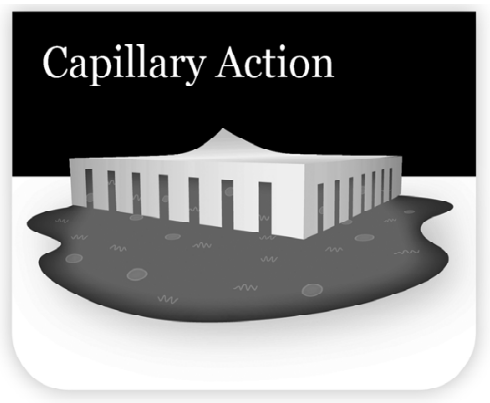

Figure 2. Capillary action caused by the attraction of molecules of the liquid (edema/exudate) to molecules of the solid (Drawtex). The small pores in the dressing act as small capillaries.

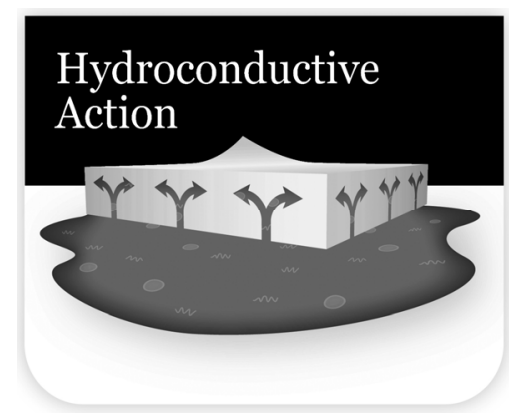

Figure 3. Hydroconductive action results from moving from wetter (wound) to drier (Drawtex) even against gravity. Governed by Darcy's Law, the fluid can move both vertically and horizontally. 
teases and the dressing. Bacteria are known to be negatively charged [11,12]. Matrix metalloproteinases such as MMP-9 are mainly negatively charged soluble proteins, although there can be some variations in electrostatic potentials within the molecules $[13,14]$. Scientists from Beier-Environtec in South Africa have recently demonstrated that Drawtex is slightly negatively charged [15]. Intuitively one would surmise that as the negatively charged bacteria and proteases approached the negatively charged dressing they would repel one another by the force of repulsion. However, that is not the case. When the negatively charged broad dressing surface is in contact with fluid, the ions from the fluid form a mobile layer of the opposite charge known as the electric double layer [12,16] (Figure 4). These mobile counter-ions (cations) are attracted to the negatively charged dressing surface. This effectively reverses the charge on the surface of the dressing to become positive, negating the force of repulsion. The more ions in the fluid, the stronger the electric double layer becomes. Serum, edema fluid, and wound exudate are full of such cations. In addition, there is an attractive force, known as Van der Waals force due to an interaction between oscillating dipoles on surface molecules [16]. Van der Waals force is a very powerful force but only operates over a very small distance. Because the broad expanse of the dressing is in intimate contact with the wound fluid, Van der Waals force can overcome any remaining repulsive force. There is a theory known as the DVLO theory that describes the interrelationship of the two main forces acting on charged particles in a solution, i.e. the electrostatic repulsion and the Van der Waals attractive force $[12,16,17]$. By combining the effect of these two forces, it is possible to describe the overall force acting to bring the negatively charged bacteria and cytokines to the surface of the dressing. The porosity of the dressing allows the substances to be drawn vertically and horizontally into the dressing by hydroconductive action as described previously.

Proof that the combination of the capillary, hydroconductive, and electrostatic result in bacteria being drawn into the Drawtex dressing can be seen using scanning electron micrographs (SEM). When Drawtex was exposed to media containing Pseudomonas aeruginosa, the bacteria were drawn into the Drawtex and trapped by the fibers (Figure 5) [18].

It appears that the combination of capillary, hydroconductive, and electrostatic actions are working in concert to provide the unique mechanistic functions attributed to Drawtex. These proposed mechanisms may not totally explain the actions of Drawtex due to the difficulty of applying physicochemical theory to biological systems [12]. The sum of these actions accounts for the effectiveness of the dressing in removing edema from the

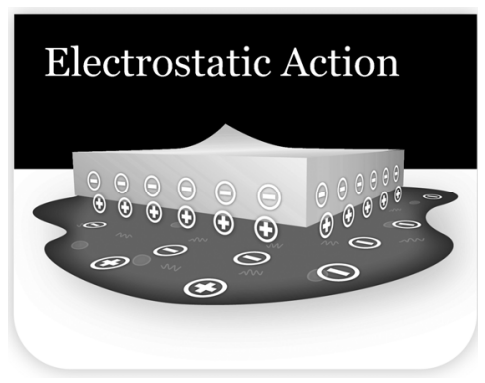

Figure 4. Electrostatic action results in attraction of negatively charged bacteria and cytokines (MMPs) to the positive charge at the surface of Drawtex. The positive charge at the Drawtex surface results from an electric double layer as positive ions from the edema/exudate coat the slightly negatively charged Drawtex.

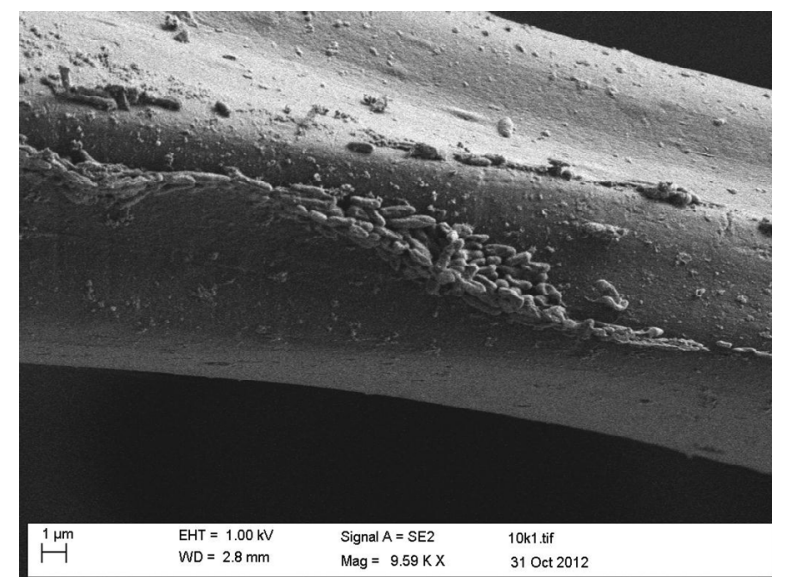

Figure 5. Drawtex immersed in a suspension with Pseudomonas aeruginosa overnight. Numerous bacteria are seen attached to the fiber of the dressing (Courtesy of Prof. Valerie Edwards-Jones, Manchester Metropolitan University).

burn wound in the clinical trial. The mechanisms of action also help to explain the results seen with the hydroconductive dressing in various types of wounds.

\section{Conclusion}

A hydroconductive dressing designed to draw off excessive wound fluid, bacteria, and deleterious cytokines can remove more wound edema fluid than standard burn gauze dressings. The target burn wounds in this study were small. It may be that if the hydroconductive dressing was used on larger TBSA wounds the amount of edema fluid in the overall burn might be decreased. Such a result would remove the deleterious effects of excess burn wound edema and help with healing of the burn wound.

\section{REFERENCES}

[1] M. C. Robson, "Advancing the Science of Wound Bed 
Preparation for Chronic Wounds,” Ostomy Wound Management, Vol. 58, No. 11, 2012, pp. 10-12.

[2] S. C. Saba, "Clinical Evaluation Comparing the Efficacy of Aquacel ${ }^{\circledR}$ Ag Hydrofiber ${ }^{\circledR}$ Dressing versus Petrolatum Gauze with Antibiotic Ointment in Partial-Thickness Burns in a Pediatric Burn Center," Journal of Burn Care \& Research, Vol. 30, No. 3, 2009, pp. 380-385. doi:10.1097/BCR.0b013e3181a2898f

[3] R. T. Ortiz, L. T. Moffatt, M. C. Robson, M. H. Jordan and J. W. Shupp, "In Vivo and in Vitro Evaluation of the Properties of Drawtex Levafiber Wound Dressing in an Infected Burn Wound Model,” Wounds, Vol. 24, No. 9, 2012, pp. 3-5.

[4] D. Ochs, M. G. Uberti, G. A. Donate, M. Abercrombie, R. J. Mannari and W. G. Payne, "Evaluation of Mechanisms of Action of a Hydroconductive Wound Dressing, Drawtex, in Chronic Wounds," Wounds, Vol. 24, No. 9, 2012, pp. 6-8.

[5] M. Wendelken, P. Lichtenstein, K. DeGroat and O. Alvarez, "Detoxification of Venous Ulcers with a Novel Hydroconductive Wound Dressing That Absorbs and Transports Chronic Wound Fluid away from the Wound," Wounds, Vol. 24, No. 9, 2012, pp. 11-13.

[6] Wikipedia, The Free Encyclopedia, "Capillary Action," 2012. En.wikipedia.org/wiki/Capillaryaction

[7] The Free Dictionary, “Capillary Action,” 2012. www.thefree-dictionary.com/capillary+action

[8] FracFocus Chemical Disclosure Registry, "Fluid Flow in the Subsurface (Darcy'sLaw)," 2012. https://www.hydraulicfracturingdisclosure.org/Account/ RegisterOperator.aspx

[9] L. Pfeiffer and C.-Y. C. Liu, "Groundwater Pumping and Spatial Externalities in Agriculture,” 2012. www.ucdavis.edu/faculty/Lin/PfeifferLin_spatial_paper.pdf
[10] “Ask Us Anything: Trees and Shrubs,” 2012. Wiki.answers.com/Q/How_does_tree_move_water_from _the_roots_to_the_top_of_the_tree

[11] A. Ljungh, N. Yanagisawa and T. Wadstrom, "Using the Principle of Hydrophobic Interaction to Bind and Remove Wound Bacteria,” Journal of Wound Care, Vol. 15, No. 4, 2006, pp. 1-6.

[12] M. Katsikogianni and Y. F. Missirlis, "Concise Review of Mechanisms of Bacterial Adhesion and of Techniques Used in Estimating Bacteria-Material Interactions,” European Cells and Materials, Vol. 8, 2004, pp. 37-57.

[13] D. G. Vartak and R. A. Gemeinhart, "Matrix Metalloproteinases: Underutilized Targets for Drug Therapy," Journal of Drug Targeting, Vol. 15, No. 9, 2007, pp. 1-20. doi:10.1080/10611860600968967

[14] B. Ganguly, J. Banerjee, A. Elegbede, D. J. Klocke, et al., "Intrinsic Selectivity in Binding of Matrix Metalloproteinase-7 to Differently Charged Lipid Membranes," FEBS Letters, Vol. 581, No. 29, 2007, pp. 5723-5726. doi:10.1016/j.febslet.2007.11.042

[15] Personal Communication from Beier Environtec.

[16] "Bite-Sized Tutorials: Electrostatic Charge and Bacterial Adhesion,” 2012. www.ncl.ac.uk/dental/oralbiol/oralenv/tutorials/electrostatic. htm

[17] W. B. Russel, D. A. Saville and W. R. Schowalter, "Colloidal Dispersions,” Cambridge University Press, New York, 1989. doi:10.1017/CBO9780511608810

[18] Personal Communication from Professor Valerie EdwardsJones, Manchester Metropolitan University, Manchester. 